\title{
A TENTATIVE CHECK LIST OF DATED SYRIAC MANUSCRIPTS UP TO 1300
}

\author{
SEBASTIAN BROCK \\ UNIVERSITY OF OXFORD
}

William Hatch's An Album of Dated Syriac Manuscripts ${ }^{1}$ has served as an invaluable guide to dated Syriac manuscripts for over half a century now, at the same time offering a wonderful resource for anyone attempting to date undated Syriac manuscripts. Hatch provided photographs of 200 manuscripts, the latest of which dates from $1593 / 4$. The number of those illustrated by him whose date is prior to 1300 is 159. Since the total number of known dated manuscripts earlier than 1300 is in fact over three times this figure, it may be helpful to offer a tentative check list of these. Once handlists and/or catalogues of the various Middle Eastern manuscript collections currently being digitized by the Hill Museum \& Manuscript Library and by other organisations are available, the present list will certainly need augmenting, especially for the twelfth and thirteenth centuries; it is hoped, nevertheless, that this preliminary list will provide a useful starting point for future studies. ${ }^{2}$

1 W.H.P. Hatch, An Album of Dated Syriac Manuscripts (Boston, 1946; repr. with an additional Foreword by L. Van Rompay, Piscataway NJ, 2002).

2 For fifth- and sixth-century manuscripts, see also S.P. Brock, 'Dating formulae in Syriac inscriptions and manuscripts of the $5^{\text {th }}$ and $6^{\text {th }}$ centuries', in a forthcoming volume edited by G. Kiraz and published by Gorgias Press. 
Details concerning catalogues of manuscripts in the different locations can be readily found in A. Desreumaux,'s Répertoire des bibliothèques et des catalogues de manuscrits syriaques (Paris, 1991); among subsequent catalogues, the following should especially be noted: J.F. Coakley, 'A Catalogue of the Syriac Manuscripts in the John Rylands Library' in Bulletin of the John Rylands Library, University of Manchester 75 (1993), 105-207; Y. Dolabani and others, 'Catalogue des manuscrits de la Bibliothèque du Patriarcat Syrien Orthodoxe à Homs (Aujourd'hui à Damas)', Parole de l'Orient 19 (1994), 555-661; F. Briquel-Chatonnet, Manuscrits syriaques de la Bibliothèque Nationale... Catalogue (Paris, 1997); I. Bcheiry, Catalogue of Syriac Manuscripts in Trinity College, Dublin (Kaslik, 2005); Philothée de Sinaï, Nouveaux manuscrits syriaques du Sinaï (Athens, 2008), and the catalogues by Dolabani and Barsaum (see under Abbreviations). ${ }^{3}$ Information concerning dated Syriac manuscripts in the collection of the Monastery of St Catherine, Sinai, is derived from H. Hussmann, 'Die syrischen Handschriften des Sinai-Klosters, Herkunft und Schreiber', Ostkirchliche Studien 24 (1975), 281-308 (some corrections have occasionally been made).

In the listing, for reasons of space, only the following very basic information is given in the five columns: I, date, including month where given; II, manuscript reference; III, illustration in Hatch's Album, where available, and (for 11 th-13 th centuries) an indication of ecclesiastical provenance; $\mathrm{IV}$, place of writing (if the scribe also gives his name, an asterisk $(*)$ is added); and V, general contents. For the abbreviations used in column V, see below.

After the listing, some annotation is provided in cases where specific comment is required; this is followed by some preliminary general observations that can be made.

The following conventions should also be noted:

${ }^{3}$ It should be noted that several of the manuscripts in Dolabany's three catalogues and in those of Barsaum are now in the Syrian Orthodox Patriarchate (and now housed in Ma'arret Saidnaya).

${ }^{4}$ For the $11^{\text {th }}$ and $12^{\text {th }}$ cent. this is only specified for E(ast Syriac) and $\mathrm{M}$ (elkite) manuscripts; all others can be assumed to be Syrian Orthodox. In the case of manuscripts of earlier date the script does not always provide a ready criterion. For East Syriac dated manuscripts, see S.P. Brock, 'Early dated Manuscripts of the Church of the East, $7^{\text {th }}$ to $13^{\text {th }}$ century', Journal of Assyrian Academic Studies 21:2 [In Honor of J.F. Coakley] (2007), 8-34. See also below, under Preliminary observations. 
- All manuscripts with 'Add.' references of five digits are in the British Library, and for convenience the relevant page number in W. Wright's Catalogue of Syriac Manuscripts in the British Museum acquired since the year 1838 (London, 1872) is given. In converting the Seleucid to the Christian era Wright did not provide the right AD date in cases where the month is given as being between October and December; in the table below, the figures he gives have been corrected wherever this is necessary (this also applies to some other catalogues, as well).

- For some libraries I have given the catalogue number rather than the shelf number, since this will be needed for any further consultation.

- In a few cases dates are given with inverted commas (e.g. '734'): these concern dates which are given wrongly in the manuscripts, or are due to misinterpretation.

- Where the date is given in more than one era, there is sometimes a lack of correspondence between the two; in these cases I have normally followed the Seleucid (AG) dating. ${ }^{5}$

- Not all manuscripts listed are still extant; this applies not only to those whose particulars are only known from information provided in subsequent copies made from them, but also to some mentioned in early modern sources which probably no longer survive.

\section{Abbreviations}

Bibl. = Biblical OT/NT; G = Gospels; L = Lectionary; Mass. = 'Massorah'). BL = British Library.

Barsaum, Srit. = A.I. Barsaum, Sritotho d-Tur 'Abdin, I-III (Ma'arrat Saydnaya, 2008- ).

${ }^{5}$ For the various eras used, see in general F. Briquel-Chatonnet, 'Le temps du copiste: notations chronologiques dans les colophons de manuscrits syriaques', in F. Briquel-Chatonnet and H. Lozachmeur (eds), Proche-Orient ancien: temps vécu, temps pensé (Antiquités sémitiques III; Paris, 1998), 197-210; and for manuscripts using dating by the Hijra era, see S.P. Brock, 'The use of Hijra dating in Syriac manuscripts: a preliminary investigation' in J.J. van Ginkel, H. Murre-van den Berg and T.M. v an Lint (eds), Redefining Christian Identity. Cultural Interaction in the Middle East since the Rise of Islam (OLA 134; 2005), 275-90. 
Dolabany, CatDZ. = F. Y. Dolabany, Catalogue of Syriac Manuscripts in Za'faran Monastery (ed. G.Y. Ibrahim; Aleppo, 1994).

Dolabany, CatJer. = F.Y. Dolabany, Catalogue of Syriac Manuscripts in St. Mark's Monastery (ed. G.Y. Ibrahim; Aleppo, 2004).

Dolabany, CatMonCh. = F. Y. Dolabany, Catalogue of Syriac Manuscripts in Syrian Churches and Monasteries (ed. G.Y. Ibrahim; Aleppo, 1994).

Géhin 2006 = 'Manuscrits sinaïtiques dispersés, I: les fragments syriaques et arabes de Paris', Oriens Christianus 90 (2006), 23-43.

Géhin $2007=$ 'Manuscrits sinaïtiques dispersés, II: les fragments théologiques syriaques de Milan (Chabot 34-57),' Oriens Christianus 91 (2007), 1-24.

Géhin $2009=$ 'Fragments patristiques syriaques des Nouvelles découvertes du Sinai', Collectanea Christiana Orientalia 6 (2009), 67-93.

Leroy $=\mathrm{J}$. Leroy, Les manuscrits syriaques à peintures, I (Paris, 1964).

Patr. G and or S = Patristic literature, Greek and/or Syriac authors

Vat. Syr. < Sinai = F. Rilliet, 'La bibliothèque de Ste-Catherine du Sinaï et ses membra disiecta: nouveaux fragments syriaques à la Bibliothèque Vaticane', VI Symposium Syriacum, OCA 247, (1994), 409-18. 


\begin{tabular}{|c|c|c|c|c|}
\hline Date & Manuscript & Hatch & Place & Content \\
\hline 411 Nov & Add. $12150=$ Cat.p.633 & I & Edessa* & Patr. G \\
\hline $459 / 60$ & Add. $14512=$ Cat. 250 & II & & Bibl. OT \\
\hline $462 \mathrm{Apr}$ & St Petersburg, Cat. no. 1 & III & $*$ & Patr. G \\
\hline $463 / 4$ & Add. $14425=$ Cat. 5 & IV & Amid* & Bibl. OT \\
\hline $473 \mathrm{Apr}$ & Vatican Syr. 160 & $\mathrm{~V}$ & & Hagiography \\
\hline 474 Sep & Add. $17182=$ Cat. 403 & VI & Edessa* & Patr. S \\
\hline $500 / 1$ & Add. $14528=$ Cat. 1030 & & Mabbug & Canon Law \\
\hline 509 Apr & Add.14542 = Cat. 417 & VII & Mon. Pa'nur* & Patr. G \\
\hline 510 Oct & Dayr al-Surian Syr. 10 & & Edessa & Bibl. NT G \\
\hline $510 / 11$ & Add.17126 = Cat. 526 & VIII & Mabbug & Patr. S \\
\hline 512 Jan & Add.17182 = Cat. 404 & IX & & Patr. S \\
\hline 518 Nov & Add.14571 = Cat. 413 & $\mathrm{X}$ & * & Patr. S \\
\hline 522 Dec & Vat. syr. 111 & XI & & Patr. S \\
\hline $528 \mathrm{Apr}$ & Vat. syr. 140 & XII & Edessa & Patr. G \\
\hline $530 / 40$ & Add $.14459=$ Cat. 68 & XIII & & Bibl. NT G \\
\hline 532 May & Add.14445 = Cat. 26 & XIV & $\begin{array}{l}\text { B.Meri, Mon. } \\
\text { Orientalis }\end{array}$ & Bibl. OT \\
\hline 532 Jun & Add.17176 = Cat. 1072 & XV & $*$ & Hagiography \\
\hline $533 / 4$ & Add.14479 = Cat. 86 & XVI & Edessa & Bibl. NT \\
\hline $533 / 4$ & Add.12175 = Cat. 637 & & & Patr. G \\
\hline 534 Jun & Sinai Syr. 46 & XVII & & Hagiography \\
\hline 535 May & Add. $14530=$ Cat. 1029 & XVIII & $\begin{array}{l}\text { Kafra d-Barta, Mon. } \\
\text { Eusebius }\end{array}$ & Canon Law \\
\hline $540 / 1$ & Add. $17107=$ Cat. 23 & XIX & Edessa & Bibl. OT \\
\hline $543 \mathrm{Dec}$ & Sinai M27N & & & Bibl. OT \\
\hline $545 \mathrm{Apr}$ & Add.14431 = Cat. 14 & & & Bibl. OT \\
\hline $548 \mathrm{Jul}$ & Vat.syr.12 & $\mathrm{XX}$ & Edessa & Bibl. NT \\
\hline $550 / 1$ & Add. $14610=$ Cat. 638 & XXI & & Patr. G \\
\hline $552 \mathrm{Feb}$ & Vat.syr.112 & XXII & Sarmin* & Patr. S \\
\hline 553 Sep & Add.12166 = Cat. 491 & XXIII & Edessa & Patr. G \\
\hline 554 Dec & Add.14635 = Cat. 414 & & & Patr. S \\
\hline $557 \mathrm{Apr}$ & Add. $14558=$ Cat. 466 & XXIV & & Patr. G \\
\hline pre 562 & Add.12156 = Cat. 648 & & & Patr. G \\
\hline 563 Aug & Vat.syr.143 & XXV & $\begin{array}{l}\text { Mon. of John of } \\
\text { Nerab }\end{array}$ & Patr. G \\
\hline $564 \mathrm{Apr}$ & Vat.syr.137 & XXVI & & Patr. S \\
\hline 564 Aug & Vat.syr.104 & XXVII & $\begin{array}{c}\text { Barbaron, Mon. } \\
\text { Quryaqos* }\end{array}$ & Patr. G \\
\hline $565 \mathrm{Feb}$ & Add. $17157=$ Cat. 505 & XXVIII & Edessa & Patr. S \\
\hline 569 May & Add.14597 = Cat. 648 & $\mathrm{XXX}$ & $\begin{array}{l}\text { Sarmin, Mon. John } \\
\text { Nerab }\end{array}$ & Patr. S G \\
\hline 569 May & Add.14599 = Cat. 547 & XXIX & Mon. B. Mar Shila* & Patr.G \\
\hline $576 \mathrm{Jul}$ & Vat.syr.142 & & Sketis & Patr.G \\
\hline 581 Jun & Add.17169 = Cat. 451 & XXXI & $*$ & Patr. S \\
\hline $581 \mathrm{Jul}$ & Vat.syr.138 & XXXII & & Patr. S \\
\hline pre 583 & Add.14464 = Cat. 70 & & Mon. Mar Zakkai & Bibl. NT G \\
\hline $584 \mathrm{Jul}$ & Add. $12160=$ Cat. 472 & XXXIII & $\begin{array}{l}\text { Mon. Gubba } \\
\text { Barraya* }\end{array}$ & Patr. G \\
\hline $586 \mathrm{Feb}$ & Florence, Laur.Plut.I.56 & XXXIV & $\begin{array}{l}\text { Mon. John, B. } \\
\text { Zagba* }\end{array}$ & Bibl. NT G \\
\hline 586 Oct & Add.14609 = Cat. 1089 & & Mon. of Speculis & Hagiography \\
\hline $587 / 8$ & Add. $12158=$ Cat .556 & & Mon. of Maki & Patr. G \\
\hline $593 \mathrm{Dec}$ & Add.17152 = Cat. 477 & XXXV & $*$ & Patr. G \\
\hline
\end{tabular}




\begin{tabular}{|c|c|c|c|c|}
\hline c.597/600 & Add.14559 = Cat. 468 & & Natpa d-Zargal & Patr. G \\
\hline $598 / 9$ & Add.17102 = Cat. 11 & XXXVI & & Bibl. OT \\
\hline $598 / 9$ & Add.14568 = Cat. 405 & & & Patr. S \\
\hline $599 / 600$ & Add. $17110=$ Cat. 118 & & Mon. of Ramsha & Bibl. OT \\
\hline $599 / 600$ & Add. $14460=$ Cat. 53 & CLX & $\begin{array}{l}\text { Tell Dinawar, } \\
\text { B.Nuhadra }\end{array}$ & Bibl. NT G \\
\hline 603 Sep & Add.14587 = Cat. 517 & XXXVII & & Patr. S \\
\hline $604 \mathrm{Jul}$ & Add. $12170=$ Cat. 458 & XXXVIII & Mathan, near Bostra & Patr. G \\
\hline 611 Sep & Add.12135 = Cat. 486 & XXXIX & $\begin{array}{l}\text { Mon. of Kawkba, } \\
\text { Hina* }\end{array}$ & Patr. G \\
\hline ‘613/4’ & (see Annotation) & XL & & \\
\hline $614 / 5$ & Add. $14471=$ Cat.54 & CLXI & Nisibis & Bibl. NT G \\
\hline 615 Aug & Paris Syr. 69 & XLI & & Patr. G \\
\hline $615 / 6$ & (Original of Harklean NT) & & $\begin{array}{l}\text { Alexandria, Mon. of } \\
\text { Ennaton }\end{array}$ & Bibl. NT \\
\hline pre 618 & Add.14567 = Cat. 479 & & $\begin{array}{l}\text { Mon. of Qashri, Mt } \\
\text { Sharda }\end{array}$ & Patr. G \\
\hline $621 / 2$ & Add. $14478=$ Cat. 91 & XLII & Haluga, near Serugh & Bibl. NT \\
\hline 624 Aug & Add. $14472=$ Cat. 82 & & Gdalta & Bibl. NT \\
\hline $633 \mathrm{Dec}$ & Assfalg, Syr.HSS, no. 5 & & Beit Hala, Damascus & Bibl. NT G \\
\hline c. 641 & Add. $14526=$ Cat. 1034 & & & Canon Law \\
\hline 653 pre & Add. $14605=$ Cat. 715 & & & Patr. G S \\
\hline $674 / 5$ & Add. $17134=$ Cat. 330 & & & Litur. H \\
\hline $681 / 2$ & Add.14666 = Cat. 92 & XLIII & & Bibl. NT \\
\hline 688 Mar & Add. $14647=$ Cat. 1094 & XLIV & * & Hagiography \\
\hline $\begin{array}{l}697 \text { Feb } \\
\text { ' } 698 \text { ' }\end{array}$ & $\begin{array}{l}\text { Add. } 12134=\text { Cat. } 29 \\
\text { (see under } 779 \text { ) }\end{array}$ & XLV & & Bibl. OT \\
\hline $699 / 700$ & Add. $14448=$ Cat. 41 & & & Bibl. NT \\
\hline 705 pre & $\begin{array}{l}\text { Dam.Patr. 12/25 } \\
\quad<\text { Jerus. } 235\end{array}$ & & $*$ & Medical \\
\hline 719 Apr & Add. $14429=$ Cat. 38 & & * & Bibl. OT \\
\hline '719/20' & Dublin, Chester Beatty 701 & CLXII & $*$ & Liturgical \\
\hline $719 / 20$ & Paris Syr. 27 & XLVII & $*$ & Bibl. OT \\
\hline 723 Apr & BL Or. 8606 & XLVIII & Edessa* & Patr. G \\
\hline 723 Oct & Dayr al-Surian Syr. 28.iv & & $*$ & Patr. G S \\
\hline 724 Mar & Add $14430=$ Cat 15 & XLIX & Mon. of Speculis* & Bibl. OT \\
\hline 726 Apr & Add. $12135=$ Cat. 25 & $\mathrm{~L}$ & Mon. of Speculis* & Bibl. OT \\
\hline ‘731-2’ & (see under $991 / 2$ ) & $\mathrm{XCV}$ & & \\
\hline '734' & (see under 834) & LI & & \\
\hline 736 Sep & Vatican Syr. 13 & LII & Urem Qastra* & Bibl. NT G \\
\hline $740 / 1$ & Berlin, Verzeichnis no.26 & LIII & & Hagiography \\
\hline $742 / 3$ & Deir al-Surian Syr. 5 & & & Bibl. OT \\
\hline $752 / 3$ & $\begin{array}{l}\text { Vorlage of Manchester, } \\
\text { Ryl. Syr. } 4\end{array}$ & & & Bibl. OT \\
\hline $756 \mathrm{Dec}$ & Florence, Laur.Plut. I.40 & LIV & Edessa & Bibl. NT G \\
\hline 758 & Sinai Syr. $38^{6}$ & & & Patr. G \\
\hline $759 / 60$ & $\begin{array}{l}\text { New York, Pierpont } \\
\quad \text { Morgan } 236\end{array}$ & & Beth Nuhadra & Bibl. NT \\
\hline $766 \mathrm{Dec}$ & $\begin{array}{l}\text { Vorlage of BL Or. } 2306 \& \\
\text { Mingana Syr. } 24\end{array}$ & & & Patr. G \\
\hline $766 / 7$ & BL Add.7157 & CLXIII & Mon. Sabrisho', & Bibl. NT \\
\hline
\end{tabular}

${ }^{6}$ See Géhin 2007, p.9; 2009, p.77. 


\begin{tabular}{|c|c|c|c|c|}
\hline & & & B.Qoqe* & \\
\hline 769 Aug & Vatican Syr. 122 & LV & & Patr. G \\
\hline 770 & Or. 8732 & LVI & $\begin{array}{l}\text { Qartmin, Mon. } \\
\text { Shem'on* }\end{array}$ & Bibl. OT \\
\hline $774 / 5$ & Add. $17170=$ Cat. 455 & LVII & Edessa* & Patr. S \\
\hline 779 Jul & Sinai Syr. 30 & & & Hagiography \\
\hline 789 Jun & Add $.17160=$ Cat .235 & LVIII & $*$ & Liturgical \\
\hline $790 \mathrm{Jul}$ & Add. $14548=$ Cat. 434 & XCVI & * & Patr. G \\
\hline 794 Jun & Mosul 94 & & & Hagiography \\
\hline 802 Mar & Add.14621 = Cat. 756 & LIX & $*$ & Patr. S \\
\hline 804 Apr & Add.12151 = Cat. 493 & LX & $*$ & Patr. G \\
\hline 806 Nov & Dolabani, CatJer, 289-96 & & $\begin{array}{l}\text { Mon. of Column, } \\
\text { Kallinikos* }\end{array}$ & Patr. S \\
\hline 815 Apr & Add.12171 = Cat. 587 & LXI & & Patr. G \\
\hline 816 Feb & Add.14582 = Cat. 692 & XCVII & $\begin{array}{l}\text { Mon. Michael, Maris, } \\
\text { Egypt* }\end{array}$ & Patr. G S \\
\hline $817 \mathrm{Jul}$ & Add. $14593=$ Cat. 590 & LXII & Edessa* & Patr. G S \\
\hline $819 / 30$ & Add.17172 = Cat. 759 & LXIII & $*$ & Patr. G S \\
\hline 822 May & Diyarbakir (Scher) 23 & & Edessa & Patr. S \\
\hline $822 / 3$ & Add.14623 = Cat. 762 & XCVIII & Thebaid* & Patr. S \\
\hline 823 Oct & Vatican Syr. 92 & XCIX & * & Liturgical \\
\hline $823 / 4$ & Add.14485 = Cat. 146 & LXIV & Harran & Bibl. L \\
\hline 824 Mar & Add.14486 = Cat. 149 & LXV & $\begin{array}{l}\text { Harran, Mon. } \\
\text { B.Qoqe }\end{array}$ & Bibl. L \\
\hline $832 / 3$ & Add.12171 = Cat. 766 & & & Patr. G \\
\hline 834 Jan & Or. 8731 & LI ('734’) & $\begin{array}{l}\text { Mon.Eusebona, B. } \\
\text { Malka* }\end{array}$ & Patr. G \\
\hline $834 / 5$ & Mingana Syr. 42C & & & Bibl. NT G \\
\hline 837 Mar & Lyon 2 & & $*$ & Patr. S \\
\hline 837 Mar & Sinai Syr. M45N & & Edessa* & Patr. S \\
\hline 837 Jun & Add.12152 = Cat. 497 & LXVI & Turlaha & Patr. G \\
\hline 839 Apr & Add.17125 = Cat. 1164 & C & Mon. Qarqaphta* & Philosophy \\
\hline 841 May & $\begin{array}{l}\text { Vorlage of Dublin, Ch. } \\
\text { Beatty Syr.3 \& Paris } \\
\text { Syr.57 }\end{array}$ & & & Bibl. NT G \\
\hline $844 / 5$ & Add.12153 = Cat. 423 & CI & Kfar Tawrata* & Patr. G \\
\hline $850 \mathrm{Apr}$ & Add.14651 = Cat. 1101 & CII & * & Hagiography \\
\hline 857 Nov & Vatican Syr. 116 & LXVIII & & Patr. S \\
\hline $858 / 9$ & Vatican Syr. 268 & LXIX & & Bibl. NT G \\
\hline 861 Mar & $\begin{array}{l}\text { Vorlage of Add.12144= } \\
\quad \text { Cat. } 912\end{array}$ & & & Patr. G S \\
\hline $861 / 2$ & Add. $14492=$ Cat. 178 & CLXIV & * & Bibl. OT L \\
\hline 866 Jan & Add.14668 = Cat. 769 & CIII & & Patr. G S \\
\hline $866 \mathrm{Apr}$ & Add. $14580=$ Cat. 767 & CIV & Edessa & Patr. G S \\
\hline$[86] 7 / 8$ & Add.12159 = Cat. 534 & $\mathrm{CV}$ & $\begin{array}{c}\text { Awlona, near } \\
\text { Antioch* }\end{array}$ & Patr. G \\
\hline $867 / 8$ & Dolabani, CatDZ, 144 & & & Bibl. NT \\
\hline 874 Aug & Dayr al-Surian Syr. 28.iii & & & Patr. G \\
\hline $873 / 4$ & Add. $17109=$ Cat 120 & LXX & Edessa & Bibl. OT \\
\hline 874 Aug & Add.17193 = Cat. 989 & & $*$ & Patr. G S \\
\hline $874 / 5$ & Add. $14650=$ Cat. 1103 & CVI & * & Hagiography \\
\hline 876 Jan & Add.12167 = Cat. 769 & CVII & & Patr. G \\
\hline $876 / 7$ & Add. $17130=$ Cat. 392 & CVIII & $*$ & Liturgical \\
\hline 882 Mar & 'Codex Syriacus Secundus' & & $\begin{array}{l}\text { Mon. of John, nr } \\
\text { Beirut* }\end{array}$ & Patr. G S \\
\hline
\end{tabular}




\begin{tabular}{|c|c|c|c|c|}
\hline $883 / 4$ & Add.18819= Cat. 340 & CIX & & Liturgical \\
\hline $885 / 6$ & Add.17194 = Cat. 1002 & $\mathrm{CX}$ & * & Patr. G S \\
\hline 886 & Vatican Syr. 623 & & Sinai* & Hagiography \\
\hline 887 Mar & Dayr al-Surian Syr. 37 & & & Liturgical \\
\hline $887 / 8$ & Add.14668 = Cat. 1196 & CXI & $\begin{array}{l}\text { Sketis, Mon. Yoldat } \\
\text { Aloho* }\end{array}$ & Patr. G S \\
\hline 893 Feb & Add. $14515=$ Cat. 240 & CXII & $\begin{array}{l}\text { Sketis, Mon. Yoldat } \\
\text { Aloho* }\end{array}$ & Liturgical \\
\hline $893 / 4$ & Paris Syr. 342 & CLXV & $\begin{array}{l}\text { Balad, Mon. of } \\
\text { Joseph* }\end{array}$ & Bibl. NT \\
\hline 899 Apr & Add.12138 = Cat. 101 & CLXVI & $\begin{array}{l}\text { Harran, Mon. of } \\
\text { Gabriel* }\end{array}$ & Bibl. Mass. \\
\hline $902 / 3$ & Or. 5021 & CXIII & Mon. Paul, Egypt* & Patr. G \\
\hline $903 / 4$ & Dayr al-Surian Syr. 30.C & & $\begin{array}{l}\text { Mon. Yoldat Aloho, } \\
\text { Sketis* }\end{array}$ & Patr. S \\
\hline 913 Mar & Add.14579 = Cat. 815 & LXXI & & Patr. G \\
\hline $916 / 7$ & Mingana Syr. 106G & & & Bibl. L \\
\hline $916 / 7$ & Sinai Syr. M3N & & & Liturgical \\
\hline $917 / 8$ & New Haven, Yale, Z107.31 & CLXVII & Mon. Elia*, Mosul & Bibl. NT G \\
\hline 927 May & Add.17111 = Cat. 125 & CXIV & $\begin{array}{l}\text { Mon. Yoldat Aloho, } \\
\text { Sketis }\end{array}$ & Bibl. OT \\
\hline $928 / 9$ & Vatican Syr. 1 & LXXII & Mosul, Mon. Elia & Bibl. OT \\
\hline $929 \mathrm{Jul}$ & Add.17174 = Cat. 1074 & CXV & & Hagiography \\
\hline 932 Apr & (Moberg, 1924) & CXVI & Qaryatein* & Hagiography \\
\hline $932 / 3^{1}$ & Sinai Syr. M15N & & Black Mt* & Hagiography \\
\hline 933 & Sinai Syr. M56N & & Sinai & Bibl. NT L \\
\hline $935 / 6$ & Add $.14469=$ Cat. 75 & LXXIII & $\begin{array}{l}\text { Sketis, Mon. Yoldat } \\
\text { Aloho* }\end{array}$ & Bibl. NT G \\
\hline $936+$ & Add.14645 = Cat. 1111 & & $\begin{array}{l}\text { Sketis, Mon. Yoldat } \\
\text { Aloho }\end{array}$ & Hagiography \\
\hline ‘938/9’ & Paris Syr. 169 & & & Liturgical \\
\hline $944 / 5$ & St Petersburg, Cat. no. 38 & & Tabriz & Medical \\
\hline $956 \mathrm{Jul}$ & Vatican Syr. 14 & LXXIV & & Bibl. NT G \\
\hline $957 \mathrm{Jul}$ & Dayr al-Suryan Syr. 22 & & Fostat & Patr. S \\
\hline $979 / 80$ & Vatican Syr, 152 & LXXV & & Bibl. Mass. \\
\hline 981 Apr & St Petersburg, Cat. no. 8 & & Nisibis & Bibl. NT \\
\hline $991 / 2$ & Harvard Syr. 176 & $\mathrm{XCV}$ & & Bibl. Mass. \\
\hline $992 / 3$ & Dam. Patr. 7/16 & & $\begin{array}{l}\text { Mon. XL Martyrs, } \\
\text { Dry River }\end{array}$ & Bibl. Mass. \\
\hline 993 Apr & Barsaum, Srit. I, 175 & & Mon. Elia, Amid(?) & Bibl. Mass. \\
\hline 994 Aug & Dam.Patr. $12 / 9<$ Jerus. 25 & LXXVI & $\begin{array}{l}\text { Melitene, Mon. XL } \\
\text { Martyrs* }\end{array}$ & Bibl. NT G \\
\hline $999 / 1000$ & Add.12139 = Cat. 154 & LXXVII & $*$ & Bibl. L \\
\hline 1000 Nov & Paris Syr. 154 & CXVII & Mon. Mar Shayna* & Liturgical \\
\hline $1000 \mathrm{Nov}$ & Damascus Patr. 12/20 & & $\begin{array}{l}\text { Mon. Sergius \& } \\
\text { Bacchus* }\end{array}$ & Patr. G S \\
\hline $1003 / 29$ & Vatican Syr. 94 & CXVIII & & Liturgical \\
\hline $1005 / 6$ & Add.12149 = Cat. 266 & & $*$ & Liturgical \\
\hline 1007 Feb & Add. $12148=$ Cat. 264 & LXXVIII & * & Liturgical \\
\hline 1014 Aug & Mosul, Mar Tuma & & Mon. XL Martyrs* & Bibl. Mass. \\
\hline 1015 Jun & Add. $12165=$ Cat. 842 & CXIX & Hisn Patriq* & Patr. G S \\
\hline 1023 Feb & Add. $14488=$ Cat. 194 & M & Black Mt* & Liturgical \\
\hline 1[0]27 Mar & Add.7158 & $\mathrm{E}$ & & Bibl. NT \\
\hline 1030 Aug & Vatican Syr. 19 [CPA] & M & $\begin{array}{l}\text { Antioch, Mon. } \\
\text { Moses }\end{array}$ & Bibl. NT L \\
\hline
\end{tabular}




\begin{tabular}{|c|c|c|c|c|}
\hline 1031 Aug & olim Leuven G151 & M & Black Mountain* & Liturgical \\
\hline 1031 Aug & Damascus Patr. 12/13 & & Mon. XL Martyrs & Patr. S \\
\hline $1033 / 4^{\circ}$ & Add. $12145=$ Cat. 251 & CXX & * & Liturgical \\
\hline 1041 Oct & Vatican Syr. 21 & M CXXI & Black Mountain* & Bibl. NT L \\
\hline $1041 \mathrm{Nov}$ & Damascus Patr. 12/21 & & Tagrit* & Bibl. NT L \\
\hline 1044 Aug & Sinai Syr. 45 & M & & Bibl. NT \\
\hline 1045 Dec & Add.14489 = Cat. 200 & $\begin{array}{l}\text { M } \\
\text { CLXXXIV }\end{array}$ & Black Mountain* & Bibl. NT L \\
\hline 1[.]49 May & Dolabany, CatJer., 154 & & Sketis? & Bibl. NT \\
\hline $1052 / 3$ & Diyarbakir (Scher) 7 & & Melitene & Bibl. NT G \\
\hline 1055 Jan & Damascus Patr. 12/8 & & Melitene* & Bibl. NT L \\
\hline $1056 \mathrm{Jul}$ & Add $.14510=$ Cat. 378 & M CXXII & Black Moutain* & Liturgical \\
\hline 1057 Jun & Sinai Syr. M5N & M & $*$ & Liturgical \\
\hline $1059 \mathrm{Apr}$ & Paris Syr. 70 & & Melitene & Liturgical \\
\hline 1059 Sep & Sinai Syr. 40 & $\mathrm{M}$ & Mon. Mar Pallad* & Liturgical \\
\hline 1061 Sep & Barsaum, Srit. I, 216 & & $\begin{array}{l}\text { Mon. XL Martyrs, } \\
\text { Dry River }\end{array}$ & Patr. S \\
\hline 1069 & Sinai Syr. 20 & M & Black Moutain & Bibl. NT L \\
\hline $1073 / 4 ?$ & Add. $17923=$ Cat. 188 & $\mathrm{E}$ & Mon. of R.Hormizd* & Bibl. NT L \\
\hline $1074 / 5$ & Add. $14714=$ Cat. 346 & CXXIII & $*$ & Liturgical \\
\hline $1078 / 9$ & Vorlage of Mingana Syr. 65 & & & Patr. S \\
\hline 1081 Mar & Add.12144 = Cat. 908 & LXXIX & $\begin{array}{c}\text { Mon.Yoldat Aloho, } \\
\text { near Alexandria }\end{array}$ & Patr. G S \\
\hline 1085 Mar & Add.14734 = Cat. 1147 & CXXIV & $\begin{array}{l}\text { Sketis, Mon. Yoldat } \\
\text { Aloho }\end{array}$ & Hagiography \\
\hline 1088 Mar & Add.17127 = Cat. 1020 & & $*$ & Patr. \\
\hline 1089 Sep & Add.14490 = Cat. 159 & LXXX & $\begin{array}{c}\text { Mon. Yoldat Aloho, } \\
\text { nr Alexandria* }\end{array}$ & Bibl. NT L \\
\hline $1092 / 3$ & Vatican, Barbarini Or.118 & & $\begin{array}{l}\text { Beth Sohroye, } \\
\text { Nineveh }\end{array}$ & Bibl. Mass. \\
\hline $1100 / 1$ & Add. $17213=$ Cat. 295 & & $\begin{array}{l}\text { Sketis, Mon. Yoldat } \\
\text { Aloho }\end{array}$ & Liturgical \\
\hline $1102 \mathrm{Jul}$ & Add.14679 = Cat. 606 & CXXV & Sketis, Niqios* & Patr. S \\
\hline $1104 \mathrm{Feb}$ & Sinai CPA 1 & CXCIX M & * & Bibl. NT L \\
\hline $1120 / 1$ & Add. $18820=$ Cat. 269 & & $*$ & Liturgical \\
\hline 1126 Nov & $\begin{array}{l}\text { Mosul (Scher) } 4 \text { (= Vorlage } \\
\text { of Vat.Borg. Syr. 113) } \\
\text { Sinai Syr. } 1\end{array}$ & & Maragha* & Bibl. OT \\
\hline 1127 Nov & Oxford, Bodl. Cat. no.164 & $\mathrm{M}$ & * & Bibl. NT L \\
\hline $1128 / 9$ & Add $.14498=$ Cat. 230 & CXXVI & & Liturgical \\
\hline 1133 Aug & Lyon 1 & CXXVII & $*$ & Liturgical \\
\hline 1138 Feb & Parus Syr. 51 & & Jerusalem* & Liturgical \\
\hline 1138 Aug & Vatican Syr. ? < Sinai & LXXXI & Jerusalem* & Bibl. NT L \\
\hline 1142 May & Dolabany, CatJer., 324-36 & $\mathrm{M}$ & $*$ & Liturgical \\
\hline 1143 Nov & Damascus Patr. $12 / 4<$ & & & Patr. S \\
\hline 1149 Sep & $\begin{array}{l}\text { Jerus. } \\
\text { Sbath Syr. } 72\end{array}$ & LXXXII & Jerusalem* & Bibl. NT L \\
\hline $1161 / 2$ & Vatican Syr. 37.ix [copy] & M & & Liturgical \\
\hline $1164 / 5$ & Paris Syr. 52 & & $\begin{array}{l}\text { Midyat, Mon. } \\
\text { Abraham* }\end{array}$ & Bibl. NT L \\
\hline $1164 / 5$ & Oxford, Bodl. Cat. no. 45 & CXXVIII & Edessa, Mon. Cross & Bibl. NT G \\
\hline 1165 Jun & Add. $14503=$ Cat. 255 & & Mon. George* & Liturgical \\
\hline 1166 Sep & Cambridge Add. 1700 & & * $\quad$ r & Liturgical \\
\hline $1169 / 70$ & Dolabani, CatJer, 179-80 & CXXIX & Edessa, Mon. Cross & Bibl. NT \\
\hline 1171 Sep & & & Hisn Ziyad* & Liturgical \\
\hline
\end{tabular}




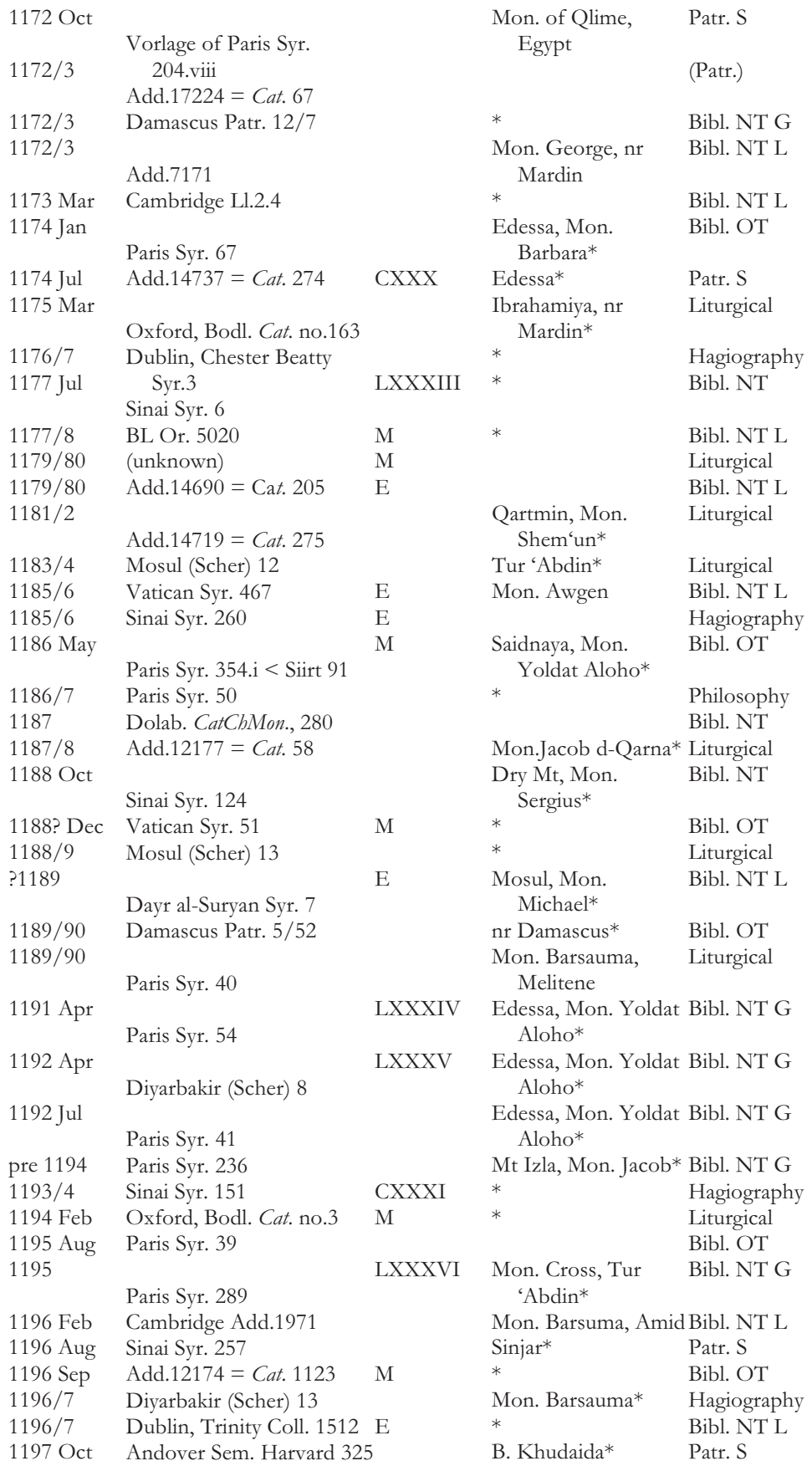




\begin{tabular}{|c|c|c|c|c|}
\hline 1198 Dec & Paris Syr. 167 & \multicolumn{2}{|c|}{ E CLXVIII } & Bibl. NT \\
\hline $1198 / 9$ & Add. $14733=$ Cat. 1139 & & Mon. Barsauma & Liturgical \\
\hline 1199 Sep & & & Sketis, Mon. Yoldat & Hagiography \\
\hline & Harvard Syr. 4 & & Aloho* & \\
\hline $1199 / 1200$ & & $\mathrm{E}$ & Mt Qardu, Mon. & Bibl. NT \\
\hline & Vosté, Cat. NDS, no.15 & & Sabrisho'* & \\
\hline $1199 / 1200$ & & $\mathrm{E}$ & Mon. Hormizd & Bibl. NT \\
\hline & 'Habouris ms.' & & & \\
\hline 1200/1(?) & Barsaum, Srit. I, 343 & $\mathrm{E}$ & Mon. Gabrona & Bibl. NT \\
\hline $1200 / 1$ & Paris Syr. 35 & W & & Bibl. NT L \\
\hline $1[2] 01 / 2$ & BL Or. 2695 & W & $\begin{array}{l}\text { Mon. Cross, Tur } \\
\text { 'Abdin* }\end{array}$ & Bibl. NT G \\
\hline $1202 / 3$ & Paris Syr. 55 & $\mathrm{E}$ & $\begin{array}{l}\text { Mon. Isho'yahb, B. } \\
\text { Nuhadra* }\end{array}$ & Bibl. NT \\
\hline $1202 / 3$ & Dolabany, CatChMon.,.300 & $\begin{array}{l}\text { W } \\
\text { LXXXVII }\end{array}$ & Mon. Elia, Amid* & Bibl. NT G \\
\hline 1203 Jan & Add. 7160 & W & & Liturgical \\
\hline 1203 Jan & Paris Syr. 31 & W & $\begin{array}{l}\text { Mon.Napshatha, } \\
\text { Edessa* }\end{array}$ & Bibl. NT \\
\hline $1203 \mathrm{Apr}$ & Barsaum, Srit. I, 185 & $\begin{array}{l}\text { W } \\
\text { LXXXVIII }\end{array}$ & Mt of Edessa* & Bibl. NT \\
\hline 1203 May & Berlin, Verz. 9 & W & Mon. Barsauma & Patr. S \\
\hline $1203 / 4$ & BL Add. 7154 & W & Mon. Abh[ai] & Bibl. NT \\
\hline $1203 / 4$ & Damascus Patr. 8/11 & W & $\begin{array}{l}\text { Mon. Yoldat Aloho, } \\
\text { Edessa* }\end{array}$ & Bibl. OT \\
\hline 1204 May & Add. 7184 & W & & Canon Law \\
\hline $1204 / 5$ & Bars. Srit. I, p.303-4 & W & $*$ & Patr. S \\
\hline $1204 / 5$ & Lund, $\mathrm{ms} 58$ & W & Mon. Qartmin & Liturgical \\
\hline $1204 / 5$ & Cambridge, BFBS 446 & W & Tagrit* & Bibl. Mass. \\
\hline $1205 / 6$ & Paris Syr. 289 & $\mathrm{E}$ & $\begin{array}{l}\text { Mon. Michael, } \\
\text { Mosul* }\end{array}$ & Bibl. NT \\
\hline 1206 Feb & BL Egerton 681 & W & Mon. Barsauma & Bibl. L \\
\hline $1206 / 7$ & (olim Neesan) & E CLXIX & Mon. Hormizd. & Bibl. NT L \\
\hline 1207 Qaita & Diyarbakir (Scher) 10 & $\mathrm{E}$ & $\begin{array}{l}\text { Mon. Michael, } \\
\text { Mosul* }\end{array}$ & Bibl. NT \\
\hline $1207 / 8$ & Harvard Syr. 26 & $\mathrm{E}$ & Mon. Hormizd* & Bibl. NT \\
\hline $1208 \mathrm{Feb}$ & Harvard Syr. 141 & W & $\begin{array}{l}\text { Mon. Yoldat Aloho, } \\
\text { Edessa* }\end{array}$ & Bibl. NT \\
\hline 1208 Jun & Vatican Syr. 78 & $\mathrm{E}$ & Mon. Hormizd* & Bibl. NT L \\
\hline $1208 \mathrm{Oct}$ & Dolabani, CatDZ, 90 & M CLXXXV & $\begin{array}{l}\text { WMon. Christopher, } \\
\text { Saidnaya }\end{array}$ & Liturgical \\
\hline $1208 / 9$ & olim Jerusalem & W & & Liturgical \\
\hline 1209 May & Harvard Syr. 14 & W & Amid* & Bibl. NT L \\
\hline $1209 / 10$ & Dolabani, CatJer.,187-9 & W & Mon.Abraham, Ingil & Bibl. NT \\
\hline $1209 / 10$ & Barsaum, Srit.I, p.265 & W & Tel Besme* & Liturgical \\
\hline 1210 Sep & Add. $17232=$ Cat. 371 & W & & Liturgical \\
\hline 1210 Sep & Vorlage of & W CXXXII & Sigistan* & Liturgical \\
\hline 1210 Sep & $\begin{array}{c}\text { Cambr.Add.1903 } \\
\text { Diyarbakir (Scher) } 11\end{array}$ & $\mathrm{~W}$ & $*$ & Bibl. NT \\
\hline $1210 / 11$ & & $\mathrm{E}$ & R. Hormizd* & Bibl. NT \\
\hline & Pierpont Morgan 235 & & & \\
\hline $1212 / 3$ & Add. $21031=$ Cat. 327 & $\mathrm{E}$ & Zirine* & Bibl. NT \\
\hline $1213 \mathrm{Feb}$ & & $\mathrm{M}$ & $*$ & Liturgical \\
\hline & Dam. Patr. 12/2 & CLXXXVI & & \\
\hline 1213 Jun & & $\mathrm{W}$ & Mon. Yoldat Aloho, & Bibl. NT \\
\hline & Vatican Syr. 82 & & Edessa* & \\
\hline
\end{tabular}


1214 Feb

1214 Jun

1214 Dec

1215 May

1215 Aug

1215 Dec

1216 Aug

1217 Nov

$1217 / 8$

$1217 / 8$

$1217 / 8$

$1217 / 8$

1218 May

1218 Oct

$1218 / 9$

1(2)19/20

1220 Mar

1220 May

1220 May

$1220 / 1$

$1220 / 1$

$1221 \mathrm{Apr}$

1221/2
1221 Sep
1222 Jan

$1222 \mathrm{Ju}$

$1222 / 3$

$1222 / 3$

$1222 / 3$

1223 Nov

$1223 / 4$

$1223 / 4$

1224 Mar

$1224 \mathrm{Jul}$

1224 Sep

$1224 / 5$

$1225 \mathrm{Ju}$

$1225 / 6$

$1225 / 6$

1226 Aug

$1226 / 7$

1228/9
Add. $18714=$ Cat. 161

M

W

Sinai Syr. 124

Sinai Syr. 234

Vatican Syr. 74

Vatican Syr. 20

Sinai Syr. 235

Dam. Patr.12/11

Paris Syr. 46

Paris Syr. 32

Cambridge, Add. 2918

Chester Beatty $4<$ Mardin W 8

Add. $17229=$ Cat .207

Charfet, Sony 11

Cambridge, Add. 1972

Chester Beatty 703

Sinai Syr. 215

BL Add. 7155

Vatican Syr. 559 [see 1260] W

Add. $14689=$ Cat. $167 \quad$ W

Vorlage of Mingana Syr. 9 W

Vorlage of Harvard Syr. W

132 and of Mingana Syr. W 559

Sinai Syr. 140

Dayr al-Surian Syr. $42 \quad$ M

Damascus Patr. $12 / 3<$ W Jerus.

Add. $14711=$ Cat. 320

Paris Syr. $365<$ Siirt 14

Add. $17253=$ Cat. 353

Add. $17922=$ Cat. 60

Paris Syr. 297

Paris Syr. 354.ii < Siirt 91

Vatican Syr. 471

Dolabani, CatChMon, 99

Vatican, Borg. Syr. 133.ii

Damascus Patr. 6/1

Vorlage of Mingana Syr. 600

Sinai Syr. 208

Paris Syr. 42

Vatican, Borg. Syr. $93 \quad$ W

Harvard Syr. 3

Mon. Mor Gabriel, Qartmin

Vatican Syr. ? < Sinai

Add. $12143=$ Cat. p.623
Mon. Christopher, Liturgical Saidnaya*

Mon. Malke, Tur Bibl. NT L 'Abdin*

Bibl. OT

Batrumin, Tripolis* Bibl. OT L

Mon. Christopher, Liturgical Saidnaya*

*

Bibl. NT L

Gangra

Mon. Cross, Tur Bibl. NT G

'Abdin

W LXXXIX Mon. Hnanya* Bibl. NT

E CLXX Mon. Gabrona* Bibl. NT

E CLXXI Mon. Jacob, B. 'Abe* Bibl. NT L

W CXXXIII* Liturgical

W Mon. Mattai* Bibl. OT

W Patr. S

W CLXII Liturgical

M Saidnaya Bibl. NT

Mon. Elia, Amid Bibl. OT

Mon. Mattai

HRSYS* Bibl. NT L

* Patr. S

Caesarea, Medical

Cappadocia*

* Liturgical

St Nicolas, Tripoli* Liturgical

Mon. Yoldat Aloho, Bibl. NT L Edessa*

M * Liturgical

CLXXXVII

E Mon. Hormizd Bibl. NT

W Mon. Yoldat Aloho, Liturgical

Sketis*

E CLXXII * Bibl. NT G

Mon. Michael, Mosul Bibl. NT G

Mon. Qayoma* Philosophy

Mon. 'Abdisho', Bibl. NT

Qom

Mon. Quryaqos Liturgical

Liturgical

Mosul* Medical

Mon. Mattai Patr. S

Mon. Thellalios, Liturgical Upper Mt*

Cairo*

Bibl. NT G

Bibl. NT L

Mon. Michael, Mosul Bibl. NT L

Mon. Jacob, Salah* Bibl. OT (for $\mathrm{Hah}$ ) 


\begin{tabular}{|c|c|c|c|c|}
\hline $1229 \mathrm{Jul}$ & Sinai Syr. 128 & W & Melitene* & Patr. S \\
\hline 1229 Aug & & M & Mon.Symeon & Bibl. OT \\
\hline & Mardin & & Styl.(Antioch)* & \\
\hline $1229 / 30$ & Add. $14691=$ Cat. 208 & W & Mon. Jacob, Salah* & Bibl. NT L \\
\hline $1230 \mathrm{Jul}$ & BL Or. 8729 & W CXXXIV & & Liturgical \\
\hline $1230 \mathrm{Jul}$ & Sinai Syr. 143 & W XCI & $\begin{array}{l}\text { Mon.Yoldat Aloho, } \\
\text { Edessa* }\end{array}$ & Bibl. NT L \\
\hline $1231 \mathrm{Jan}$ & Paris Syr. 402 & M & & Bibl. OT \\
\hline $1231 / 2$ & Sinai Syr. 81 & W & Mt of Edessa* & Bibl. NT \\
\hline $1232 / 3$ & Sinai Syr. 121 & M & Mon. Elia, Antioch* & Bibl. NT L \\
\hline $1232 / 3$ & Add. $17124=$ Cat. 42 & M & $*$ & Bibl. OT \\
\hline $1233 / 4$ & Ancestor of Mingana & W & Mon. Natpa* & Bibl. NT \\
\hline $1233 / 4$ & $\begin{array}{c}\text { Syr. } 174 \\
\text { Sinai Syr. } 261\end{array}$ & W & Mon.Antony & Hagiography \\
\hline $1233 / 4$ & Vatican Syr. 464 & M & Sinai* & Liturgical \\
\hline $1233 / 4$ & Vatican Syr. 147 & $\mathrm{~W}$ & $*$ & Patr. S \\
\hline 1234 Oct & Paris Syr. $359<$ Mardin 46 & W CXXXV & $\begin{array}{l}\text { Mon. Barsauma, nr } \\
\text { Mardin }\end{array}$ & Patr. S \\
\hline pre 1235 & Sinai Syr. 146 & $\mathrm{E}$ & Walton* & Patr. S \\
\hline $1235 \mathrm{Mar}$ & Sinai Syr. 112 & M & & Liturgical \\
\hline $1235 / 6$ & Sinai Syr. 103 & M & * & Bibl. OT \\
\hline $1236 \mathrm{Feb}$ & Vatican Syr. 80 & M & $*$ & Bibl. OT \\
\hline 1236 Aug & Add. $14678=$ Cat. 132 & $\begin{array}{l}\text { M CL- } \\
\text { XXXVIII }\end{array}$ & Saidnaya & Liturgical \\
\hline $1236 / 7$ & Damascus, Syr. Cath. Ch. & W & $\begin{array}{l}\text { Mon. Yoldat Aloho, } \\
\text { Sketis }\end{array}$ & Bibl. OT \\
\hline $1236 / 7$ & Sinai Syr. 77 & M & & Liturgical \\
\hline $1237 \mathrm{Jul}$ & Chaldean Patr. 1225 & M & * & Liturgical \\
\hline $1237 / 8$ & Oxford, Bodl. Cat. no 65 & $\mathrm{E}$ & Baghdad* & Bibl. NT L \\
\hline 1238 Aug & Paris Syr. 112 & W & $\begin{array}{l}\text { Mon. Yoldat Aloho, } \\
\text { Edessa* }\end{array}$ & Liturgical \\
\hline $1238 / 9$ & Siirt 114 & W & & Liturgical \\
\hline $1239 \mathrm{Feb}$ & Add. $17246=$ Cat. 307 & W & $*$ & Patr. S \\
\hline 1239 Mar & Diyarbakir 57 & W & $\begin{array}{l}\text { Mon. Yoldat Aloho, } \\
\text { Sketis }\end{array}$ & Liturgical \\
\hline $1240 \mathrm{Feb}$ & Sinai Syr. 258 & $\mathrm{E}$ & $*$ & Liturgical \\
\hline 1240 Jun & Berlin, Verz. no. 15 & W & $\begin{array}{l}\text { Mon.Yoldat Aloho, } \\
\text { Sketis* }\end{array}$ & Liturgical \\
\hline $1240 / 1$ & Sinai Syr. 256 & W & Mon. Jacob, Salah* & Bibl. NT L \\
\hline $1240 / 1$ & Sinai Syr. 242 & $\mathrm{E}$ & $*$ & Liturgical \\
\hline 1241 Aug & Add. $21210=$ Cat. 876 & $\mathrm{M}$ & Mon. Christopher & Bibl. OT \\
\hline 1242 May & Sinai Syr. 111 & W CXXXV] & I Mosul* & Patr. S \\
\hline 1242 Dec & Sinai Syr. 131 & M & $\begin{array}{l}\text { Mon.Yoldat Aloho, } \\
\text { Black Mountain* }\end{array}$ & Liturgical \\
\hline $1242 \mathrm{Dec}$ & St Petersburg, Cat. no. 22 & M & $*$ & Liturgical \\
\hline 1243 Sep & Vorlage, Tehran Issayi 18 & $\mathrm{E}$ & Urmi & Bibl. NT L \\
\hline $1243 / 4$ (?) & Assfalg, Verz. 6.ii & $\mathrm{E}$ & & Patr. S \\
\hline 1244 Jun & BL Or. 5265 & W & & Bibl. NT \\
\hline $1244 / 5$ & Oxford, Bodl. Cat. no. 32 & $\mathrm{E}$ & & Bibl. NT \\
\hline 1245 Nov & Vatican Syr. 194 & W & Mon. John, Sketis* & Bibl. NT G \\
\hline 1246 Dec & Vatican, Borgia Syr. 159 & $\mathrm{E}$ & Baghdad* & Patr. S \\
\hline pre 1247 & Add. $17257 \stackrel{\circ}{=}$ Cat. 141 & W & Qal 'at Romayta(?)* & Liturgical \\
\hline $1247 / 8$ & Berlin, Ver₹. no. 25 & W & $\begin{array}{l}\text { Mon. Yoldat Aloho, } \\
\text { Sketis* }\end{array}$ & Bibl. OT \\
\hline 1248 May & Vatican $<$ Sinai & W & $\begin{array}{c}\text { Mon. Lazarus, } \\
\text { Habsenas* }\end{array}$ & Liturgical \\
\hline
\end{tabular}




\begin{tabular}{|c|c|c|c|c|}
\hline 1248 Nov & BL Or. 2299 & M & Sinai* & Liturgical? \\
\hline $1248 / 9$ & Mosul (Scher) 71 & $\mathrm{E}$ & $*$ & Liturgical \\
\hline $1248 / 9$ & Jerusalem,Patr.Gr. 37 & $\mathrm{E}$ & * & \\
\hline $1249 / 50$ & Barsaum, Srit. III, 438 & $\mathrm{E}$ & & Liturgical \\
\hline $1249 / 50$ & Add. $17256=$ Cat. 142 & W & Mon. Abai & \\
\hline 1251 Sep & Sinai Syr. 233 & W XCII & $\begin{array}{l}\text { Mon. Yoldat Aloho, } \\
\text { Sketis* }\end{array}$ & Bibl. OT \\
\hline 1252 Jan & Paris Syr. 367.ii < Siirt 29 & M & Sinai* & Liturgical \\
\hline 1252 Jan & Vatican Syr. 81 & $\mathrm{E}$ & $\begin{array}{l}\text { Mon. Sabrisho', B. } \\
\text { Qoqa* }\end{array}$ & Patr. S \\
\hline 1252 Sep & Add. $17227=$ Cat. 94 & M & Sinai* & Liturgical \\
\hline $1253 / 4$ & Sinai Syr. 88 & W & $\begin{array}{l}\text { Mon. John the Small, } \\
\text { Sketis* }\end{array}$ & Bibl. NT \\
\hline 1254 Feb & Barsaum, Srit, 1, 336 & M & Sinai* & Liturgical \\
\hline $1254 \mathrm{Oct}$ & Sinai Syr. 155 & W & Mon. Jacob d-Qarna & Liturgical \\
\hline $1254 / 5$ & Sinai Syr. 25 & M & & Bibl. OT \\
\hline 1255 Mar & Sinai Syr. 64 & M & Sinai* & Liturgical \\
\hline 1255 Jun & Add. $14686=$ Cat. 170 & M & Sinai* & Liturgical \\
\hline 1255 Sep & Sinai Syr. 129 & W XCIII & $\begin{array}{l}\text { Mon. Yoldat Aloho, } \\
\text { Sketis* }\end{array}$ & Bibl. L \\
\hline $1255 / 6$ & Paris Syr 134 & M & $*$ & Liturgical \\
\hline $1255 / 6$ & Sinai Syr. 212 & M & $\begin{array}{l}\text { Mon. Yoldat Aloho, } \\
\text { Kaftun }\end{array}$ & Liturgical \\
\hline $1256 \mathrm{Jan}$ & Add. $14687=$ Cat. 172 & M & $*$ & Liturgical \\
\hline 1256 Jan & Add. $14715=$ Cat. 380 & W & $\begin{array}{l}\text { Mon. Yoldat Aloho, } \\
\text { Sketis* }\end{array}$ & Bibl. L \\
\hline $1256 / 7$ & Sinai Syr. 85 & W & $\begin{array}{l}\text { Mon. Yoldat Aloho, } \\
\text { Sketis* }\end{array}$ & Liturgical \\
\hline 1257 Jan & Sinai Syr. 237 & M & Sinai* & Liturgical \\
\hline $1258 \mathrm{Jul}$ & Add. $14710=$ Cat. 325 & M & Sinai* & Liturgical \\
\hline $1258 / 9$ & Sinai Syr. 78 & $\begin{array}{l}\text { M } \\
\text { CLXXXIX }\end{array}$ & * & Liturgical \\
\hline 1259 Oct & Sinai Syr. 87 & M & $*$ & Liturgical \\
\hline $1259 \mathrm{Nov}$ & Berlin, Verz. no. 88 & M & Sinai* & Liturgical \\
\hline $1259 / 60$ & Vatican $<$ Sinai & E CLXXIII & & Philosophy \\
\hline $1259 / 60$ & Sinai Syr. 157 & M & Antioch & \\
\hline $1260 \mathrm{Feb}$ & Sinai Syr. 159 & M & Sinai* & Bibl. OT \\
\hline $1260 \mathrm{Feb}$ & Sinai Syr. 98 & M & Sinai* & Bibl. OT \\
\hline $1260 \mathrm{Apr}$ & Vatican Syr. 559 & $\mathrm{M}$ & & Bibl. OT \\
\hline 1260 May & Jerusalem, Greek Patr. 9 & W & Mon. Mattai & Bibl. NT L \\
\hline $1260 / 1$ & Sinai Syr. 220 & & $\begin{array}{l}\text { Mon. George, nr } \\
\text { Aleppo }\end{array}$ & Bibl. NT \\
\hline $1261 \mathrm{Apr}$ & Sinai Syr. 165 & M & Sinai* & Liturgical \\
\hline $1261 \mathrm{Apr}$ & Vatican Syr. 11 & M & * & Bibl. OT \\
\hline $1261 \mathrm{Nov}$ & Vorlage of St Petersburg, & M & $*$ & Bibl. OT \\
\hline $1261 / 2$ & $\begin{array}{l}\text { Cat. 41, Mosul (Scher) } \\
\text { 26, and Urmia } 218\end{array}$ & $\mathrm{E}$ & Tabriz* & Patr. S \\
\hline $1262 \mathrm{Nov}$ & $\begin{array}{l}\text { Dam. Patr. } 12 / 12< \\
\text { Jerusalem } \\
\text { Add. } 14701=\text { Cat. } 278\end{array}$ & W & & Bibl. NT L \\
\hline $1262 / 3$ & Paris Syr. 56 & W & & Liturgical \\
\hline $1264 \mathrm{Jul}$ & & W & Mon. Mattai* & Bibl. NT G \\
\hline & Sinai Syr. 90 & CXXXVII & & \\
\hline 1265 Aug & Cambridge, Add. 1967 & M & Sinai* & Liturgical \\
\hline $1265 / 6$ & Vatican Syr. 59 & $\mathrm{E}$ & & Bibl. NT \\
\hline 1266 Mar & Sinai Syr. 166 & W Maron. & & Liturgical \\
\hline
\end{tabular}




\begin{tabular}{|c|c|c|c|c|}
\hline $1266 / 7$ & Sinai Syr. 222 & $\mathrm{M}$ & Sinai & Liturgical \\
\hline $1266 / 7$ & Vatican Syr. 148 & $\mathrm{M}$ & Sinai* & Bibl. NT L \\
\hline 1267 Jul & BL Or. 3336 & $\mathrm{E}$ & $*$ & Patr. S \\
\hline $12[6] 8 \mathrm{Jul}$ & BL Add. 7189 & $\mathrm{E}$ & Mon.Basima, Qardu & Patr. S \\
\hline $1268 / 9$ & Sinai Syr. 255 & W & Mon. Mattai* & Patr. S \\
\hline 1271 Jun & Sinai M71N & M & $\begin{array}{l}\text { Mon. Jacob, nr } \\
\text { Batroun* }\end{array}$ & Liturgical \\
\hline 1272? Dec & Dolabani, CatDZ, 141-3 & M & Sinai & Liturgical \\
\hline $1272 / 3$ & Sinai Syr. 245 & W & Hisn Ziyad* & Bibl. NT L \\
\hline 1274 Jun & (not extant) & M & $\begin{array}{l}\text { Mon. Yoldat Aloho, } \\
\text { Kaftun* }\end{array}$ & Bibl. OT \\
\hline 1275 & Berlin, Verz. no. 138 & W & $*$ & Patr. S \\
\hline 1275 Sep & Vorlage of Vatican Syr. 66 & W & Hisn Ziyad* & Bibl. NT \\
\hline $1275 / 6$ & Harvard Syr. 27 & $\mathrm{E}$ & Hamadan* & Liturgical \\
\hline 1276 Jan & Cambridge, Add. 2003 & $\mathrm{E}$ & $\begin{array}{l}\text { Mon. Yonan, Piroz } \\
\text { Shabur* }\end{array}$ & Bibl. NT \\
\hline 1276 May & Dayr al-Surian Syr. 39 & $\begin{array}{l}\text { W } \\
\text { CXXXVIII }\end{array}$ & $*$ & Patr. S \\
\hline 1276 Jun & Vat. Borgia Syr. 133.II & W & & Liturgical \\
\hline 1277 Jul & Dolabani, CatJer., 247 & W & & Liturgical \\
\hline 1277 Aug & Florence, Laur. 230 & W & & Liturgical \\
\hline 1278 Aug & Sinai Syr. 253 & W & * & Patr. S \\
\hline 1278 Aug & Sinai Syr. 158 & M & $\begin{array}{l}\text { BSha'le, Mt } \\
\text { Lebanon* }\end{array}$ & Bibl. OT \\
\hline 1278 Aug & Florence, Laur. 86 & $\mathrm{M}$ & $\begin{array}{l}\text { Mon. Tuma al- } \\
\text { Zabdani* }\end{array}$ & Liturgical \\
\hline $1278 \mathrm{Dec}$ & Paris Syr. 155 & W & & Patr. S \\
\hline $1278 / 9$ & Berlin, Verz. no. 151 & W & Efkosia, Cyprus & Liturgical \\
\hline $1279 / 80$ & (not extant) & W & * & Liturgical \\
\hline $1279 / 80$ & Dolabani, CatJer., 218 & $\mathrm{E}$ & $\begin{array}{l}\text { Mon. Michael, } \\
\text { Mosul* }\end{array}$ & Bibl. NT \\
\hline $1281 / 2$ & Sinai Syr. M19N & W & & Liturgical \\
\hline $1281 / 2$ & Sinai Syr 116 & M & $\begin{array}{l}\text { Mon. Paximata, } \\
\text { Cilicia }\end{array}$ & Bibl. NT L \\
\hline $1282 \mathrm{Nov}$ & Vatican, Borgia Syr. 132 & $\mathrm{M}$ & Sinai* & Liturgical \\
\hline $1283 / 4$ & Add. $17236=$ Cat. 318 & W & Mon. Mattai & Patr. S \\
\hline 1284 Apr & Sinai Syr. 156 & M CXC & $\begin{array}{l}\text { Mon. Yoldat Aloho, } \\
\text { Kaftun* }\end{array}$ & Liturgical \\
\hline 1284 Sep & Vorlage of Vatican Borg. & M & Sinai & Liturgical \\
\hline $1284 / 5$ & $\begin{array}{l}\text { Syr. } 169 \\
\text { Vorlage of Mingana Syr. }\end{array}$ & $\mathrm{E}$ & Sinjar & Bibl. NT L \\
\hline 1285 Aug & $\begin{array}{l}310 \\
\text { Sinai Syr. } 91\end{array}$ & W & Mosul & Patr. S \\
\hline 1286 Aug & Sinai Syr. 123 & M & Sinai* & Liturgical \\
\hline 1286 Oct & Kirkuk (Vosté) 1 & $\mathrm{M}$ & & Liturgical \\
\hline $1286 / 7$ & Mardin (Scher) 22 & $\mathrm{E}$ & $\begin{array}{l}\text { Mon. Michael, Kfar } \\
\text { 'Uzail }\end{array}$ & \\
\hline 1287 Dec & Sinai X9N & $\mathrm{E}$ & Mon. Augen* & Liturgical \\
\hline $1287 / 8$ & Sinai Syr. 271 & M & & Liturgical \\
\hline $1288 \mathrm{Feb}$ & Sinai Syr. 207 & M & Sinai* & Bibl. NT L \\
\hline 1288 May & Jerusalem, Greek Patr. 34 & M & Sinai* & Liturgical \\
\hline 1288 Oct & BL Add. 7173 & $\mathrm{E}$ & & Patr. S \\
\hline $1288 / 9$ & & $\mathrm{E}$ & $\operatorname{Artok}(*)$ & Bibl. NT L \\
\hline 1289 May & $\begin{array}{l}\text { Alqosh } 237=\text { Chald.Mon. } \\
\text { Baghdad } 680 \\
\text { Sinai Syr. } 272\end{array}$ & $\begin{array}{l}\text { CLXXIV } \\
\text { E }\end{array}$ & Mon. Hormizd & Patr. S \\
\hline
\end{tabular}




\begin{tabular}{|c|c|c|c|c|}
\hline 1289 Aug & Pampakuda 33 & $\mathrm{M}$ & Sinai* & Bibl. NT G \\
\hline $1290 \mathrm{Jan}$ & Sinai Syr. 203 & W & & Patr. S \\
\hline $1290 \mathrm{Feb}$ & Trichur 64 & M & Sinai* & Liturgical \\
\hline 1291 May & Vatican, Borgia Syr. 146 & $\mathrm{E}$ & * & Patr. S \\
\hline 1291 Sep & Jerusalem, Mon. St Mark & W & & Patr. S \\
\hline 1291 Oct & $\begin{array}{l}208 \\
\text { Mingana Syr. } 81\end{array}$ & W & & Patr. S \\
\hline $1291 / 2$ & Add. $14699=$ Cat. 304 & M & * & Patr. S \\
\hline $1292 \mathrm{Jul}$ & Sinai Syr. 92 & $\begin{array}{l}\text { W } \\
\text { CXXXIX }\end{array}$ & $\begin{array}{l}\text { Mon. Yoldat Aloho, } \\
\text { Sketis* }\end{array}$ & Liturgical \\
\hline $1292 \mathrm{Sep}$ & Vat. $<$ Sinai & M & Sinai* & Bibl. NT \\
\hline $1292 \mathrm{Sep}$ & Mosul (Scher) $99=$ & M & Sinai* & \\
\hline $1292 \mathrm{Dec}$ & $\begin{array}{l}\text { Vorlage of Mingana Syr. } \\
156 \\
\text { Sinai Svr. } 83\end{array}$ & & Maragha* & Patr. S \\
\hline $1293 \mathrm{Mar}$ & Sinai Syr. 227 & M & * & Liturgical \\
\hline $1293 \mathrm{Jul}$ & Sinai Syr. 236 & M & Sinai* & Bibl. NT \\
\hline 1294 Mar & Karamlais 1 & M & Sinai* & Bibl. NT L \\
\hline $1294 / 5$ & Sinai Syr. 210 & $\mathrm{E}$ & Karamlais* & \\
\hline $1295 \mathrm{Mar}$ & Sinai Syr. 75 & M & Sinai* & Liturgical \\
\hline $1295 \mathrm{Sep}$ & Sinai Syr. 95 & M & Sinai* & Bibl. NT \\
\hline $1295 \mathrm{Sep}$ & Sinai Syr. 217 & M & Sinai* & Liturgical \\
\hline $1296 / 7^{1}$ & Sinai Syr. 80 & M & Sinai* & Bibl. NT L \\
\hline $1297 \mathrm{Apr}$ & Berlin, Ver: no. 182 & M & Sinai* & Liturgical \\
\hline $1297 / 8$ & Vorlage of Berlin, Verz. no. & & Mon. Mattai & Patr. S \\
\hline $1297 / 8$ & $\begin{array}{l}83 \text { and Mingana Syr. } 212 \\
\text { Charfet, Sony } 181\end{array}$ & & Khlat* & Patr. S \\
\hline $1297 / 8$ & Paris Syr. 335 & W & Manzikert* & Patr. S \\
\hline pre 1298 & Vatican Syr $622<$ Diyarb. & W? & & Bibl. NT \\
\hline $1298 \mathrm{Mar}$ & $\begin{array}{c}9 \\
\text { Dublin, Trinity Coll. } 1504\end{array}$ & $\mathrm{E}$ & * & Bibl. NT G \\
\hline $1298 \mathrm{Dec}$ & Vorlage of Mingana Syr. 47 & $\mathrm{WW}$ & * & Patr. S \\
\hline $1298 / 9$ & Sinai Syr. 169 & W & & Canon Law \\
\hline $1298 / 9$ & Chicago A 11997 & M & Sinai & Liturgical \\
\hline $1299 \mathrm{Dec}$ & Urmi 194 & W & & Patr. S \\
\hline $1299 / 1300$ & Dolabani, CatJer., 237 & $\mathrm{E}$ & & Liturgical \\
\hline $1300 \mathrm{Mar}$ & & W & Gozarto d-Qardu & Liturgical \\
\hline
\end{tabular}

\section{Annotation}

534 Jun Sinai Syr. 46: see Géhin 2007: 15.

586 For this famous manuscript, see now M. Bernabò (ed.), Il Tetravangelo di Rabbula (Rome, 2008).

'613/4' Hatch XL: Milan, Ambrosiana: Hatch's date is incorrect, and the ms. is probably $8^{\text {th }}$ cent.; see Géhin 2006: 15, and his 'Reconstitution et datation d'un recueil syriaque melkite (Ambr.A 296 inf., ff.222-224 + Sinai Syr. 10)', Rivista di Studi Bizantini e Neoellenici 42 (2005), 51-68.

705 pre Damascus Patr. 12/25: Various dates in the early eighth century have been given for this important medical 
manuscript; for the date given here, see G. Kessel, 'The Syriac Epidemics (Damascus, Syriac Orthodox Patriarchate $12 / 25$ ) and the problem of its identification', forthcoming.

'719/20' Chester Beatty 701: although the colophon indeed gives AG 1031 (= AD 719/20), the scribe must have omitted the number for the hundreds, for the hand cannot be so early; furthermore, pace Hatch, the manuscript is quite a developed West Syriac Fenqitho, which implies a date several centuries later. To judge by the script illustrated in Hatch, it would seem most likely that the date should have been AG $1<5>31$, i.e. AD 1219/20.

723 Apr BL Or. 8606: for the colophon, see R.W. Thomson, 'An eighth-century Melkite colophon from Edessa', Journal of Theological Studies ns 13 (1962), 249-58; cp also Géhin 2007: 14.

758/9 Sinai Syr. 38: see Géhin 2007:9 and 2009: 77.

759/60 Pierpont Morgan 236: The colophon gives AG 1061 and AH 142; that the latter must be the correct one is indicated by the mention of 'Mar Jacob, Catholicos Patriarch of the Church of the East', who reigned from 753-73. (The colophon also mentions Ephrem, bishop of Beth Nuhadra who is otherwise unrecorded).

779 Jul Sinai Syr. 30: for this date (rather than 698, as given in Hatch, XLVI, and elsewhere), see S.P. Brock, 'Syriac on Sinai: the main connections', in V. Ruggieri and L. Pieralli (eds), Eukosmia. Studi miscellanei per il 750 di Vincenzo Poggi S.J. (Soveria Mannelli, 2003), 106-7, note 16.

794 Jun Mosul 94: For this manuscript see R. Draguet, Formes syriaques de la matière de l'bistoire Lausiaque, I (CSCO Scr. Syri 169; 1978), 47*-63*, 83*-93* (the ms. number is there given as 801, ex 94).

834 BL Or. 8731: the date is given incorrectly as AG 1045, rather than 1145; see A. de Halleux, 'Rabban Benjamin d'Édesse et la date du ms. B.L. Or. 8731', IV Symposium Syriacum 1984 (OCA 229, 1987), 445-51. 
837 Mar Sinai M45N: this is part of the famous manuscript of Sahdona, nothing of which had hitherto been known as still being in St Catherine's Monastery; see Géhin 2007:19-20 and S.P. Brock, 'New fragments of Sahdona's Book of Perfection at St Catherine's Monastery, Mount Sinai', Orientalia Christiana Periodica 75 (2009), 175-8.

841 May See P. Kahle, 'The Chester Beatty manuscript of the Harklean Gospels', in Miscellanea G. Mercati 6 (Studi e Testi 126; 1946), 208-33.

867/8 Evidently once in Midyat; Dolabani gives no further information. Might this possibly have been the 'manuscript on parchment of the ninth century, containing a transliteration of the Greek Gospels in the Syriac letters', which O. H. Parry saw in Midyat, according to his Six Months in a Syriac Monastery (London, 1895), 211?

882 Mar 'Codex Syriacus Secundus': for this ms., originally belonging to St Catherine's Monastery, Sinai, see W. Strothmann, Codex Syriacus Secundus (Göttingen: Orientforschungen, Reihe Syriaca 13; 1977).

886 Vatican Syr. 623: see Géhin, 2006: 33-4.

902/3 BL Or. 5021: see S.P. Brock, 'Notulae Syriacae', Le Muséon 108 (1995), 74-6, and Géhin 2009: 84-5, who identifies this as part of Sinai M54N.

917/8 See L. Depuydt, 'Classical Syriac manuscripts in Yale University Library', Hugoye 9:2 (2006).

$928 / 9$ For the problem over the date, see S.P. Brock in Journal of Assyrian Academic Studies 21:2 (2007), 20.

932 Apr For this ms., which consists of folios recovered from a binding, see A. Moberg, The Book of the Himyarites (Lund, 1924); the manuscript was in a private collection in Sweden, but was on sale in London in 2007.

932/3 Sinai M15N: see Géhin 2009: 75-6.

936+ Add. 14645: The date of 936 which Wright gives for the manuscript in fact refers to the date of the translation, from Arabic into Syriac, of the Life of John Colobos; since the copyist describes the circumstances of this, it is 
likely that he was writing shortly after 936; see S.P. Brock, 'Abbot Mushe of Nisibis, collector of Syriac manuscripts', in E. Vergani (ed.), (Milan), forthcoming.

'938/9' Paris Syr. 169: as Zotenberg observed in his Catalogue, 'la date est fausse'.

991/2 Harvard Syr. 176: for this date, rather than 732 in Hatch XCV, see J.F. Coakley, The Typography of Syriac (London, 2006), pp.9-10.

994 Aug Damascus Patr. 12/9: for a description, see also Dolabany, CatJer., 129-32.

1000 Nov Paris Syr. 154: cp also F. Nau, Journal Asiatique XI.5 (1915), 534-5.

1000 Nov Dam. Patr. 12/20: see Dolabany, CatDZ., 23-37, and A. Vööbus, Handschriftliche Überlieferung der memreDichtung des Ja'qob von Serug (CSCO Subs 39; 1973), 958.

1014 Aug Mosul, Mar Tuma: see J. Leroy, Les manuscrits syriaques à peintures, I (Paris, 1964), 221.

1015 Jun Add. 12165: cp also A. N. Palmer, Oriens Christianus 70 (1986), 47.

1055 Jan Dam. Patr. 12/8: cp Leroy, Les manuscrits syriaques à peintures, I, 225-33.

1073/4? Add. 17923: see S.P. Brock in Journal of Assyrian Academic Studies 21:2 (2007), 22.

1126 Nov Mosul 4: the ms. is now Baghdad, Chaldean Patriarchate, 211: see R.J.V. Hiebert, The 'Syrobexaplaric' Psalter (Atlanta, 1989), 8-9.

1031 Aug olim Leuven G 151: this is one of the Sinai manuscripts from Hiersemann Katalog 500 (1922), no. 34, which was destroyed in the Second World War.

1031 Aug Dam. Patr. 12/13: see Vööbus, Handschriftliche Überlieferung, I, 138-41; Dolabany, CatDZ., 59-71.

1059 Apr Paris Syr/70: cp also F. Nau, Journal asiatique XI.5 (1915), 492-5.

1092/3 Vatican, Barbarini Or. 118: cp Leroy, Les manuscrits syriaques à peintures, I, 236-7. 
$1138 \mathrm{Feb} \quad$ Lyon 1: for a translation of the long colophon, see A.N. Palmer, Oriens Christianus 76 (1992), 77-82.

1138 Aug Paris Syr. 51: for a translation of the colophon, see Palmer, Oriens Christianus 76 (1992), 82-5.

1149 Sep Dam. Patr. 12/4: for a translation of the long colophon, see Palmer, Oriens Christianus 76 (1992), 8590.

1177 Jul Dublin, Chester Beatty 3: for the colophons, see P.E. Kahle, 'The Chester Beatty Manuscript of the Harklean Gospels', in Studi e Testi 126 (1946), 208-33.

1180 For a report of this ms. see the Quarterly Report of the Assyrian Mission XXVII (1897), 147-9. It was written 'in the time of Patr. Elia and Mar Sabrisho' of Mergi and Amadya'.

1188/9 Vatican Syr. 51: see H. Kaufhold, 'Über Datum und Schreiber der Handschrift Vaticanus Syriacus 51', Aram 5 (1993), 267-75.

1200/1 'Habouris ms.': The final digit in the Seleucid date is unclear, and might be either (AG) 1512 or 1519 (AD $1200 / 1$ or $1207 / 8$; I was able to see the manuscript briefly before it was auctioned at Sotheby's London in July 1986).

1200/1 Barsaum, Srit. I, 343, where he gives AG 1512 for this illuminated manuscript from 'Ainwardo, and this should be accepted, rather than the date given by $\mathrm{P}$. Harb, 'Unbekannte Handschriften in Tur 'Abdin', in III Symposium Syriacum (OCA 221; 1983), 350.

1204 May Damascus Patr. 8/11: this is the West Syriac collection of Canons published by A. Vööbus, The Synodicon in the West Syrian Tradition I-II (CSCO Scr. Syri 161-4; 1975).

1205/6 Cambridge, BFBS 446: this can be identified as the manuscript described, by I.H. Hall, 'On a manuscript of the Peshitto New Testament, with the Traditions of the Apostles', Journal of the American Oriental Society 14 (1890), Proceedings, lix-lxxxv.

1207 Qaita For this manuscript, dated AG 1518, 2nd Sunday of Qaita, see R. H. Gottheil, 'On a Syriac manuscript of 
the New Testament belonging to the Rev. Mr Neesan', Journal of the American Oriental Society 13 (1889), Proceedings, clxxxi-clxxxxiii.

1209 May This manuscript was seen by A. Rücker on sale in Jerusalem in 1926: see his 'Bericht über einiger syrische Handschriften', Oriens Christianus III.2 (1927), 159-63.

1213 Jun See also Dolabany, CatDZ, 11-14; Barsawm, Srit. II, 42-57.

1217 Nov Dam. Patr. 12/11: see also Barsawm, Srit. II, 58-61.

1(2)19/20 Chester Beatty 701: see annotation to '719/20' for the revised dating.

1222 Jan Dam. Patr. 12/3: see Leroy, Les manuscrits syriaques à peintures I, 313- 20; Dolabany, CatJer., 144-9.

1226/7 For this illustrated Gospel Lectionary, written for Hah, see Leroy, Les manuscrits syriaques à peintures I, 321-32; Dolabany, CatDZ, 143; and Barsaum, Srit. I, 132. (The AD date given by Harb, III Symp. Syr., 352, is incorrect).

$1229 / 30 \quad$ For this illuminated manuscript, see the detailed description by I. Gülten in Qolo Suryoyo 13 (1980), 2422 [sic]; also in brief Barsaum, Srit. I, 133 (vid.), ZA and in his History of Tur 'Abdin (Glane/Losser, 1985), 133.

1236/7 Damascus: for this Euchologion, see Baumstark, 'Syrische und syro-arabische Handschriften in Damaskus', Oriens Christianus 5 (1905), no. 49.

1237/8 Chald. Patr. Baghdad 1225: for this manuscript, see JM. Fiey, 'Résidences et sépultures des patriarches syriaques orientaux', Le Muséon 98 (1985), 160.

1238 Aug For the illuminations, see L. Doumato, in Cabiers archéologiques 52 (2005/8), 57-61.

1243/4 For this manuscript, see A. Desreumaux, Apocrypha 5 (1994), 144.

1260 May Vat. Syr. 559. The AG date can be read as either 1531 or 1571, depending on whether the ambiguous third digit is read as a lamadh $(=30)$, or as an ' $e(=70)$. 
Since the day of the week is given (Saturday), and the 'beginning' of the month May, the reading 1571 is probably to be preferred, since 1 May 1260 was indeed a Saturday. For the later date, but based on different criteria, see J.M. Fiey, 'Iconographie syriaque: Hulagu, Doquz Khatun... et six ambons?', Le Muséon 88 (1975), 59-68.

$1267 \mathrm{Jul} \quad$ Vat. Syr. 148: See also P-G. Borbone, Egitto e Vicino Oriente 26 (2003), 74.

$1268 \mathrm{Jul}$ BL Or. 3336: The digit indicating the decade is damaged, and could be read as [arb]'in or [tesh]' $i n$, as well as [shab]' in (i.e. AD 1238, 1288 are also possibilities); see S.P. Brock, Hugoye 6:2 (2009), 198.

1272 Sinai M71N. The scribe gives 'one thousand, seven hundred, eight hundred'! In Mother Philothea's catalogue this is taken as AM 6780, and converted to AD 1272, using the standard Byzantine world era; since some other Sinai manuscripts use the Alexandrian world era, the date by this would be AD 1288.

1272/3 Dolabany lists nine illuminations; the artist is Dioscorus, known from other illuminated manuscripts (e.g. Leroy, I, 371-83).

1278 Aug Florence Or. 230. For this manuscript, see also A. Sawma, Gregory Barhebraeus' Commentary on the Book of Kings from his Storehouse of Mysteries (Uppsala, 2003), 132-3.

1279/80 (not extant): according to a note in an East Syriac ms. in St. Mark's Monastery, Jerusalem, this manuscript was copied by 'Abdisho' of Soba himself, and was seen in the Monastery of St Michael (Mosul): see the translation by H. Kaufhold, Introduction to I. Perczel (ed.), The Nomocanon of Metropolitan Abdisho of Nisibis (Piscataway, 2005), xiii.

1284/5 For other descendants of this ms., see Kaufhold, Introduction to Perczel (ed.), The Nomocanon, xiii.

1285 Aug The scribe of Mingana syr. 310 states that the original was in Bar 'Ebroyo's own hand, and was dated 22 
Aug AG 1596 (= AD 1285), in Mosul (August is the month given by Mingana, but see $H$. Takahashi, Barhebraeus, A Bio-Bibliography (Piscataway, 2005), 252 who gives ' $11^{\text {th }}$ Teshri I', i.e. October (see also p.251, on Damascus, Patr. 6/2, said to date from 1286).

1288/9 BL Add. 7173. For the date, see Wright, Catalogue, 1204.

1289 May The contents of this manuscript are given by J-M. Vosté, 'Recueil d'auteurs ascétiques nestoriens du VIIe et VIIIe siècle’, Angelicum 6 (1929), 143-206.

1290 Jan Pampakuda 33. For this manuscript of Bar 'Ebroyo's Nomocanon, see H. Kaufhold, Syrische Handschriften juristischen Inhalts in südindischen Bibliotheken (Österr. Akad. Wiss., phil.-hist. Kl., Sb. 535 Band; Wien, 1989), 28-30.

1291 May Trichur 64. For this manuscript of "Abdisho"s Nomocanon, see H. Kaufhold, Syrische Handschriften juristischen Inbalts, 38-40, and his Introduction to the photographic reproduction, ed. I. Perczel, The Nomocanon of Metropolitan Abdisho of Nisibis (Piscataway, 2005).

1298 Mar Vat. syr. 622. For this manuscript, on blue paper, see P-G. Borbone, 'I Vangeli per la principessa Sara', Egitto e Vicino Oriente 26 (2003), 63-82.

\section{Preliminary ObServations}

First of all, it is instructive to note the comparative figures for each century:

$\begin{array}{lllll}\text { Century } & \text { No. of Mss } & \text { Syr. Orth. } & \text { Ch. East } & \text { Melk. } \\ \text { Fifth } & 6 & & & \\ \text { Sixth } & 45 & & & \\ \text { Seventh } & 16 & & & \\ \text { Eighth } & 23 & & & \\ \text { Ninth } & 45 & & & \\ \text { Tenth } & 26 & 21 & 2 & 9 \\ \text { Elevnth } & 32 & 52 & 8 & 9 \\ \text { Twelfth } & 64 & 107 & 49 & 79 \\ \text { Thirteenth } & 235 & & \end{array}$


The most striking feature here lies in the figures for the thirteenth century, significantly the time of the Syriac 'renaissance', which by far outnumber those of the other centuries; in this connection it should be noted that for the twelfth century, 52 out of the total 64 belong to the last four decades of that century; in other words, they too belong to the period of the Syriac 'renaissance'. The earlier part of the twelfth century, by contrast, belongs to one of the thinnest periods, with no representatives at all in the second and sixth decade of that century. The only other decades for which no dated manuscripts are attested are the sixth and seventh decades of the seventh century, and the seventh and eighth decades of the tenth century. By contrast, the sixth and ninth centuries are remarkably well represented; this is of especial significance as far as the sixth century is concerned, given the fact that many texts ceased to be copied, or copied in full, after about the eighth century. ${ }^{7}$

It is only from the eleventh century onwards that script is normally a reliable guide to ecclesiastical origin. For the previous centuries it is only contents and/or specific information provided by the colophon that can be used as a safe criterion. On these grounds the following can be safely identified as either Melkite or belonging to the Church of the East (there is only a single dated Maronite manuscript, of 1266, that survives from the period under consideration):

Church of the East (14 mss): ${ }^{8} 599 / 600,614 / 5,682,699 / 700$, 759/60, 766/7, 794, 861/2, 893/4, 899, 917/8, 928/9, 944/5, 981.

Melkite (9 mss): 723(Apr), 779, 817, 837 (Sinai Syr.), 882, 886, 917, 932/3, 933.

As for places where manuscripts were copied, ${ }^{9}$ it is remarkable that Edessa is so well represented, despite complete gaps for the

7 For this, see especially S.P. Brock, 'Without Mushe of Nisibis, where would we be? Some reflections on the transmission of Syriac literature', in R. Y. Ebied and H. Teule (eds), VIIIth Symposium Syriacum = Journal of Eastern Christian Studies 56 (2004), 15-24.

${ }^{8}$ For these, see in more detail S.P. Brock, 'Early dated manuscripts of the Church of the East, $7^{\text {th }}$ to $13^{\text {th }}$ century' [see note 4].

9 In the following, since the place is indicated in the list of dated manuscripts above, for manuscripts dated by the Seleucid era, only the year for Jan. to Sep. is given. 
tenth, eleventh and first half of the twelfth century; those for the late twelfth and thirteenth century were almost all written in monasteries on the 'Mountain of Edessa'. Apart from two Melkite manuscripts $(723,837)$, all Edessene manuscripts are of Syrian Orthodox provenance:

Edessa (33 mss): 411, 474, 510, 528, 534, 541, 548, 553, 565, 723, 756, 775, 817, 822, 837, 866, 874, 1165, 1170, 1174(two), 1191, 1192(two), 1203(two), 1204, 1208, 1213, 1222, 1230, 1232, 1238.

Given that it has been the Dayr al-Suryan that has preserved a very high proportion of the surviving dated manuscripts of the fifth to thirteenth century, it is not surprising that it (or, in a few cases, another Egyptian monastery) has such a high number of manuscripts written there or elsewhere in Egypt:

Egypt (32 mss): 576, 816, 823, 888, 893, 903, 904, 927, 936, 936+, 957, 1049(?), 1081, 1085, 1101, 1102, 1172, 1199, 1223, $1225,1234,1237,1239,1240,1245,1247,1251,1254,1255$, $1256,1257,1292$.

Also well represented are monasteries in the Melitene area, among which are four $(1197,1199,1203,1206)$ from the famous Monastery of Barsaumo):

Melitene area (15 mss): 993, 994, 1014, 1031, 1053, 1055, 1059, 1061, 1188, 1190, 1197, 1199, 1203, 1206, 1229.

Another famous monastery, that of Mar Mattai in north Iraq, is also quite well attested, all in the thirteenth century:

Mar Mattai (8 mss): 1218, 1220, 1225, 1260, 1264, 1269, 1284, 1298.

A certain number of manuscripts originate from monasteries in Tur 'Abdin:

Qartmin (Mor Gabriel Monastery) (3 mss): 770, 1182, 1205.

Other Tur 'Abdin monasteries (14 mss): 1165, 1184, 1188, 1194, 1195, 1204, 1202, 1214, 1217, 1227, 1230, 1241, 1248. 1254.

Of the East Syriac monasteries, two are quite well represented:

Mar Michael (6 mss): 1189, 1206, 1207. 1223, 1226, 1280

Rabban Hormizd (8 mss): 1074, 1200, 1207, 1208(two), 1211, 1223, 1289. 
Some other famous monasteries are less well represented: Sabrisho': 767, 1200, 1252; Elia: 918, 929; Awgin: 1186, 1287; Beth 'Abe: 1218.

Since it is St Catherine's Monastery, Sinai, which has preserved the vast majority of surviving Melkite manuscripts, it is not surprising that no less than 34 were written there in the course of the thirteenth century, mostly from the second half of that century, and above all during its last two decades. Though 837 was written specifically for the monastery (then, Mar Mushe), the only manuscript prior to the thirteenth century written there was $886 .{ }^{10}$

An important scribal tradition, not only in Syriac, was located in monasteries on the Black Mountain, to the north west of Antioch (8 mss):11 933, 1023, 1031, 1041, 1045, 1056, 1069, 1242. A further important Melkite scribal centre was Saidnaya (7 mss): $1186,1208,1214,1215,1220,1236,1241$; later, near the end of the thirteenth century, several scribes working on Sinai state that they came from Saidnaya $(1291,1292,1293,1295)$. Of some significance is the presence of three Melkite manuscripts written in the Monastery of the Yoldat Aloho in Kaftun $(1256,1274,1284) .{ }^{12}$

It is also instructive to observe the changing contents of the manuscripts over the centuries. Employing the general categories of Biblical, Patristic (Syriac), Patristic (Greek), Hagiography, Liturgical, Canon Law, Medicine and Philosophy, we find the following figures (the total number of dated manuscripts is also indicated for each century):

10 For early Syriac connections with Sinai, see further S.P. Brock, 'Syriac on Sinai: the main connections', in V. Ruggieri and L. Pieralli (eds), Eukosmia. Studi miscellanei per il 750 di Vincenzo Poggi S.J. (Soveria Mannelli, 2003), 103-17.

${ }^{11}$ For manuscripts written on the Black Mountain, see S.P. Brock in R. Schulz and M Görg (eds), Lingua Restituta Orientalis. Festgabe für J. Assfalg (Aegypten und Altes Testament 20; 1990), 59-67.

12 For their interest, see R. Jabre Mouawad, 'Les mystériaux monastères de Keftun au Liban à l'époque médiévale (XII-XIIe s.): maronite et puis melkite?', Tempora 12/13 (2001/2), 95-113. In recent years some important wall-paintings have been uncovered in Kaftun: see M. Immerzeel, Identity Puzzles. Medieval Christian Art in Syria and Lebanon (Orientalia Lovaniensia Analecta 184; 2009), 94-9. 


\begin{tabular}{|c|c|c|c|c|c|c|c|}
\hline $\begin{array}{l}\text { Century } \\
+ \text { total } \\
\text { mss }\end{array}$ & Biblical & $\begin{array}{l}\text { Patr. } \\
\text { Syr. }\end{array}$ & $\begin{array}{l}\text { Patr. } \\
\text { Gk }\end{array}$ & Hagiogr. & Liturgical & $\begin{array}{l}\text { Canon } \\
\text { Law. }\end{array}$ & $\begin{array}{l}\text { Med. } \\
\text { Philos- } \\
\text { ophy }\end{array}$ \\
\hline $5^{\text {th }} 6$ & 2 & 1 & 2 & 1 & - & & - \\
\hline $6^{\text {th }} 45$ & 13 & 13 & 17 & 3 & - & 2 & - \\
\hline 7th 16 & 8 & 3 & 6 & 1 & 1 & 1 & - \\
\hline $8^{\text {th }} 23$ & 10 & 3 & 7 & 3 & 2 & - & 1 \\
\hline $9^{\text {th }} 45$ & 10 & 27 & 29 & 3 & 5 & - & 1 \\
\hline $10^{\text {th }} 26$ & 14 & 2 & 2 & 4 & 2 & - & 1 \\
\hline $11^{\text {th }} 32$ & 14 & 10 & 7 & 1 & 12 & - & - \\
\hline $12^{\text {th }} 64$ & 34 & 4 & 1 & 5 & 19 & - & 1 \\
\hline $13^{\text {th }} 234$ & 97 & 37 & - & 1 & 84 & 1 & 3 \\
\hline
\end{tabular}

\section{SOME NOTABLE TRENDS}

Although dated manuscripts will constitute only a small proportion of the total number of surviving manuscripts for any given century, they are probably sufficient in number to allow us to observe certain general trends. ${ }^{13}$ Here, attention might be drawn to the following ones.

Biblical manuscripts provide a high proportion for each century, with half (or over) of the total number for the century in the seventh, tenth and twelfth century. Lectionary manuscripts first appear in the ninth century, and there is a notable cluster of 'masoretic' manuscripts in the late tenth and early eleventh century.

Up to the ninth century translations of Greek patristic writers preponderate over native Syriac authors, but in the ninth century they are nearly equally balanced. In the following centuries Greek writers are much less well represented, and disappear entirely in the thirteenth century. Manuscripts combining both Greek and Syriac authors are poorly represented except in the ninth century (when there are ten such manuscripts); this combination is completely absent from the twelfth and thirteenth centuries. Although Syriac authors continue to be copied, with a quite high number in the thirteenth century, it is noticeable that, with the exception of Jacob of Serugh, these primarily concern writers of the ninth century and later.

Liturgy became increasingly common from the ninth century onwards; in the eleventh and twelfth centuries liturgical

${ }^{13}$ See also the general remarks in 'The Art of the Scribe', chapter 8 in S.P. Brock and D.G.K. Taylor (eds), The Hidden Pearl, II. The Heirs of the Ancient Aramaic Heritage (Rome, 2001), 243-62, esp. 247-9. 
manuscripts constitute approximately a third of the total for those centuries, while for the thirteenth century they are well over a third.

The numbers for the remaining categories are too low to discern any significant trends. It can be noted that, from the eleventh century onwards, the Melkite manuscripts are all either biblical (usually psalters) or liturgical.

Finally, it is interesting to note the different months in which manuscripts were completed (often, of course, the copying will have run over several months). ${ }^{14}$ The figures for the period up to 1300 are as follows:

\begin{tabular}{|c|c|c|c|}
\hline & Teshri I (Oct.): & 16 & Nisan (Apr.): \\
\hline & Teshri II (Nov): & 18 & Iyyar (May): \\
\hline & Kanun I (Dec.): & 21 & Hziran (Jun.): \\
\hline & Kanun II (Jan): & 17 & Tammuz (Jul.): \\
\hline & Shbat (Feb.): & 24 & $\mathrm{Ab}$ (Aug.): \\
\hline & Adar (Mar.): & 26 & Illul (Sep.): \\
\hline Total & Winter months: & 122 & Summer months \\
\hline
\end{tabular}

Not surprisingly the total for the summer months, when daylight was significantly longer, is higher. What is surprising is the low figure for June (and comparatively low one for May); the significance of this is not (to me, at least) clear.

In conclusion it needs to be recollected that, especially once catalogues for several important Middle Eastern libraries become available, the present tentative checklist will undoubtedly need to be expanded, above all as far as the later centuries are concerned; nevertheless it is hoped that the present summary assemblage of materials will serve as a helpful initial basis for further studies.

14 This information is occasionally given by the scribe; a very informative modern colophon detailing this is to be found in the late Mor Julius Çiçek's beautifully calligraphed Gospel Lectionary, published in reproduced form by the Bar Hebraeus Verlag in 1987. 